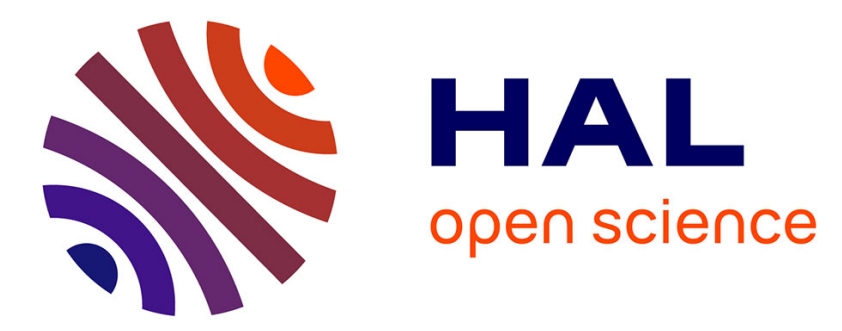

\title{
Microbial community structure reveals instability of nutritional symbiosis during the evolutionary radiation of Amblyomma ticks
}

Florian Binetruy, Marie Buysse, Quentin Lejarre, Roxanne Barosi, Manon Villa, Nil Rahola, Christophe Paupy, Diego Ayala, Olivier Duron

\section{To cite this version:}

Florian Binetruy, Marie Buysse, Quentin Lejarre, Roxanne Barosi, Manon Villa, et al.. Microbial community structure reveals instability of nutritional symbiosis during the evolutionary radiation of Amblyomma ticks. Molecular Ecology, 2020, 29 (5), pp.1016-1029. 10.1111/mec.15373 . hal03001756

\section{HAL Id: hal-03001756 https://hal.science/hal-03001756}

Submitted on 18 Nov 2020

HAL is a multi-disciplinary open access archive for the deposit and dissemination of scientific research documents, whether they are published or not. The documents may come from teaching and research institutions in France or abroad, or from public or private research centers.
L'archive ouverte pluridisciplinaire HAL, est destinée au dépôt et à la diffusion de documents scientifiques de niveau recherche, publiés ou non, émanant des établissements d'enseignement et de recherche français ou étrangers, des laboratoires publics ou privés. 
1 Microbial community structure reveals instability of nutritional symbiosis

2 during evolutionary radiation of Amblyomma ticks

3 Florian Binetruy ${ }^{\mathrm{a} *}$, Marie Buysse ${ }^{\mathrm{a}}$, Quentin Lejarre ${ }^{\mathrm{a}, \mathrm{b}}$, Roxanne Barosi ${ }^{\mathrm{a}}$, Manon Villa ${ }^{\mathrm{a}}$, Nil

$4 \quad$ Rahola $^{\mathrm{a}, \mathrm{b}}$, Christophe Paupy ${ }^{\mathrm{a}}$, Diego Ayala ${ }^{\mathrm{a}, \mathrm{b}}$ and Olivier Duron ${ }^{\mathrm{a}}$

5

6 a MIVEGEC (Maladies Infectieuses et Vecteurs : Ecologie, Génétique, Evolution et

7 Contrôle), Centre National de la Recherche Scientifique (CNRS) - Institut pour la Recherche

8 et le Développement (IRD) - Université de Montpellier (UM), Montpellier, France

$9{ }^{\mathrm{b}}$ CIRMF (Centre International de Recherches Médicales de Franceville), Franceville, Gabon

$10 *$ Correspondance: florian.binetruy@ird.fr 


\section{Abstract}

Mutualistic interactions with microbes have facilitated the adaptation of major eukaryotic lineages to restricted diet niches. Hence, ticks with their strictly blood-feeding lifestyle are associated with intracellular bacterial symbionts through an essential B vitamin supplementation. In this study, examination of the whole bacterial diversity in 25 tick species of the Amblyomma genus showed that three intracellular bacteria, Coxiella-like endosymbionts (LE), Francisella-LE and Rickettsia, are remarkably common. No other bacterium is so uniformly present in Amblyomma ticks. Almost all Amblyomma species were found to harbour a nutritive obligate symbiont, Coxiella-LE or Francisella-LE, able to synthesize B vitamins. However, despite the co-evolved and obligate nature of these mutualistic interactions, the structure of microbiomes does not mirror the Amblyomma phylogeny with a clear exclusion pattern between Coxiella-LE and Francisella-LE across tick species. Coxiella-LE, but not Francisella-LE, form evolutionarily stable associations with ticks commonly leading to co-cladogenesis. We further evidenced symbiont replacements during radiation of Amblyomma, with recent, and likely ongoing, invasions by Francisella-LE and subsequent replacements of ancestral Coxiella-LE through transient co-infections.

Nutritional symbiosis in Amblyomma ticks is thus not a stable evolutionary state, but instead arises from conflicting origins between unrelated but competing symbionts with similar metabolic capabilities. 


\section{Introduction}

Macro-organisms harbour complex microbial communities living inside and on their body (Margulis, 1993; Theis et al., 2016). These microbial communities, known as the microbiomes, can determine pivotal phenotypic traits of their hosts, driving a variety of ecological and evolutionary processes including major nutritive, reproductive and immune functions (Gould et al., 2018; Groussin et al., 2017; Hanning \& Diaz-Sanchez, 2015; Ley et al., 2008; Turner, James, \& Poole, 2013). As hosts vary in the microbiomes they harbour, an associated functionally important phenotypic variation exists within host populations (Falony et al., 2016; Ferrari \& Vavre, 2011; Jaenike, 2012; Oliver, Russell, Moran, \& Hunter, 2003; Scarborough, Ferrari, \& Godfray, 2005). In arthropods, these microbiomes notably include highly specialized intracellular bacteria depending almost exclusively on maternal (transovarial) transmission to ensure their persistence in host populations (Moran, McCutcheon, \& Nakabachi, 2008; Wernegreen, 2012). Some of these maternally inherited symbionts are essential for the life cycle of their arthropod hosts: They are obligate mutualists able to synthesize biochemical products favouring the specialization of arthropods to novel habitats or to particular feeding niches such as strict haematophagy or phloemophagy (Moran et al., 2008; Wernegreen, 2012). Overall, these mutualistic interactions have facilitated the radiation of major arthropod lineages, leading to remarkable host-symbiont phylogenetic congruence with a strict co-cladogenesis pattern in many cases (Chen, Li, \& Aksoy, 1999; Duron et al., 2017; Jousselin, Desdevises, \& Coeur d'acier, 2009; Moran, Tran, \& Gerardo, 2005; Takiya, Tran, Dietrich, \& Moran, 2006).

Arthropods and beneficial maternally inherited symbionts can form evolutionary stable associations lasting for millions of years, but that are not necessarily permanent (Bennett \& Moran, 2015; McCutcheon, Boyd, \& Dale, 2019; Moran et al., 2008; Wernegreen, 2012). 
Recent phylogenetic reconstructions suggest that beneficial symbiotic relationships can break down: Recently acquired symbionts can replace ancestral beneficial symbionts and provide similar benefits to the host (McCutcheon et al., 2019; Sudakaran, Kost, \& Kaltenpoth, 2017). An alternative scenario is that recently acquired symbionts may cooperate with ancestral beneficial symbionts (Moran et al. 2008; Vautrin \& Vavre, 2009). Vertical transmission actually locks the different symbionts together as coinfection and creates then priviliged situations for symbiont-symbiont interactions, especially cooperation and dependence between symbionts. Functions of ancestral beneficial symbionts may be complemented by recently acquired cosymbionts and their coexistence can be ultimately stable over millions of years (Meseguer et al 2017). New beneficial symbionts often originate from microbes abundant in the host environment, potentially including entomopathogens, parasites vectored by arthropods or other maternally inherited symbionts, primarily facultative (i.e. not essential) for host survival (Koga \& Moran, 2014; Matsuura et al., 2018; McCutcheon et al., 2019; Sachs, Skophammer, \& Regus, 2011). These facultative symbionts, however, determine important traits in arthropods: protection against natural enemies, adaptation to changing environments or reproductive traits (Engelstädter \& Hurst, 2009; Ferrari \& Vavre, 2011; Moran et al., 2008; Oliver et al., 2003). Contrary to beneficial obligate symbionts, facultative symbionts undergo occasional horizontal transfers (HT) across arthropod species, resulting in limited phylogenetic congruence between hosts and symbionts (Duron, Wilkes, \& Hurst, 2010; Jousselin, Cœur d'Acier, Vanlerberghe-Masutti, \& Duron, 2013; Russell et al., 2009). Overall, the diverse range of microbial lifestyle strategies creates a complex web of interactions mediating the dynamics of beneficial symbioses in arthropods (McCutcheon et al., 2019). 
Co-existence of symbionts within microbial communities is expected to involve interactions ranging from cooperation to competition and that can, in turn, determine aggregation and exclusion patterns (Ferrari \& Vavre, 2011; Moran et al., 2008; Vautrin \& Vavre, 2009).

Exclusion patterns have been recently detected between maternally inherited symbionts of ticks, suggesting that replacements of beneficial symbionts occur in this system (Duron et al., 2017). Among arthropods, ticks (Arachnida: Ixodidae) are well known to engage in symbiotic associations with at least 10 different genera of maternally inherited bacteria (Ahantarig, Trinachartvanit, Baimai, \& Grubhoffer, 2013; Duron et al., 2017). Ticks are specialized for an exclusive diet of vertebrate blood, and have evolved intimate interactions with beneficial symbionts that provide essential B vitamins and co-factors deficient in the blood diet (Bonnet, Binetruy, Hernández-Jarguín, \& Duron, 2017; Duron et al., 2017, 2018; Gerhart, Moses, \& Raghavan, 2016; Gottlieb, Lalzar, \& Klasson, 2015; Guizzo et al., 2017; Hunter et al., 2015; Olivieri et al., 2019; Smith, Driscoll, Gillespie, \& Raghavan, 2015). Approximately two thirds of tick species harbour Coxiella-like endosymbionts (Coxiella-LE hereafter), which are required for tick survival and reproduction (Gottlieb et al., 2015; Guizzo et al., 2017; Smith et al., 2015; Zhong, Jasinskas, \& Barbour, 2007). Coxiella-LE genomes encode pathways for the biosynthesis of major B vitamins and co-factors that fit closely with the expected nutritional complements required for strict haematophagy (Gottlieb et al., 2015; Guizzo et al., 2017; Smith et al., 2015). Coxiella-LE are abundant in two organs of ticks: ovaries, that is consistent with vertical transmission into developing oocytes, and Malpighian tubules, where B vitamins are possibly synthesized (Buysse, Plantard, McCoy, Duron, \& Menard, 2019; Wang et al., 2018)(Lalzar et al 2012). Owing to their maternal inheritance and beneficial nature, Coxiella-LE are present in most individuals within infected host species [16, 41]. In the Rhipicephalus tick genus, the acquisition of Coxiella-LE was followed by co- 
diversification resulting in deeply congruent Rhipicephalus-Coxiella-LE phylogenies (Duron et al., 2017).

In a few tick species, however, Coxiella-LE are present at much lower frequencies than expected for obligate nutritional symbionts, suggesting that they are instead facultative symbionts in these hosts (Duron et al., 2015). Phylogenetic evidence corroborates this hypothesis, since closely related Coxiella-LE may infect distantly related tick species suggesting recurrent HT of some Coxiella-LE (Duron et al., 2015). In other tick species, no Coxiella-LE were detected at all, but alternative obligate beneficial symbionts have been identified or hypothesized (Duron et al., 2017, 2018; Gerhart et al., 2016; Kurtti et al., 2015; Olivieri et al., 2019). Indeed, Francisella-like endosymbionts (Francisella-LE) are commonly found in tick species lacking Coxiella-LE. A recent analysis of endosymbiotic communities in 81 tick species also showed that there is a significant exclusion pattern between FrancisellaLE and Coxiella-LE (Duron et al., 2017). Like Coxiella-LE, Francisella-LE are essential for tick nutrition: Ticks deprived of their Francisella-LE completely cease development but resume normal growth upon supplementation with B vitamins (Duron et al., 2018). Genomes of Francisella-LE contain roughly the same biosynthesis pathways of B vitamins and cofactors as observed in Coxiella-LE genomes (Duron et al., 2018; Gerhart et al., 2016). Francisella-LE also presents the same tropism than Coxiella-LE: Francisella-LE are abundant in ovaries and Malpighian tubules of the ticks they infect (Duron et al 2018, current biology). Although Francisella-LE and Coxiella-LE are distantly related, they have converged towards an analogous nutritional mutualism with ticks [32, 33].

While Coxiella-LE symbioses are likely ancestral in ticks, replacements by Francisella-LE having recently transitioned to an endosymbiotic lifestyle (Duron et al., 2018; Gerhart et al., 
2016) appear across the tick phylogeny (Duron et al., 2017). Yet, the factors favouring

Francisella-LE over Coxiella-LE in this evolutionary dynamic are not well understood. In this study, we examined the evolutionary dynamic of tick microbiomes, with a focus on beneficial nutritional symbioses, in an ecologically diverse genus of hard ticks, Amblyomma. This genus is the third largest in the family Ixodidae, with its species primarily occupying the tropical zones. The centre of species diversity is on the American continent, where half of all the 130 Amblyomma species are found (Guglielmone, Estrada-Peña, Keirans, \& Robbins, 2003). The Amblyomma genus includes major vectors of tick-borne disease agents, including the lone star tick, A. americanum, which is the primary vector of Ehrlichia spp. (Childs \& Paddock, 2003). Only a few studies have examined the microbial diversity in Amblyomma species, showing that they are infected either by Coxiella-LE or by Francisella-LE in addition to other maternally inherited bacteria (Binetruy, Dupraz, Buysse, \& Duron, 2019; Budachetri et al., 2014; Clay et al., 2008; Duron et al., 2017; Gerhart et al., 2016). Here, we investigated the variation in tick microbial communities at different geographic and phylogenetic scales using a representative collection of specimens covering ca. 20\% of Amblyomma species diversity. First, we reconstructed the Amblyomma phylogeny through the sequencing of large nuclear rDNA sequences (18S, ITS1, 5.8S, ITS2, and 28S rDNA). Second, we extensively characterized bacterial communities in Amblyomma species through a DNA barcoding approach targeting the $16 \mathrm{~S}$ rDNA. Third, we traced the evolutionary histories of Coxiella-LE and Francisella-LE using multilocus sequence typing (MLST) systems. While the CoxiellaLE MLST already exists (Duron et al., 2015), in this study we developed a specific Francisella-LE MLST. Finally, we compared Amblyomma phylogeny with microbiome structure and further used co-phylogenetics, by comparing Amblyomma, Coxiella-LE and Francisella-LE phylogenies, to reveal the global dynamics of symbiotic interactions. 
Tick collection and processing

We examined a total of 144 tick specimens belonging to 25 Amblyomma species (1-11

specimens per species) collected from field sites in America and Africa or from laboratory colonies (Supplementary Table S1). Samples were preserved in $70 \%$ ethanol until use. To eliminate external (i.e. cuticular) microbes, tick specimens were surface cleaned with bleach prior to DNA extraction (Binetruy, Dupraz, et al., 2019). A few specimens (A. loculosum, n=4; A. sculptum, $n=1$; Amblyomma sp., $n=1$, obtained from a previous study (Duron et al., 2017)) were, however, not bleach-treated prior to DNA extraction (Supplementary Table S1). Hilden, Germany).

Molecular typing of ticks

To reconstruct Amblyomma phylogeny, we sequenced almost complete rDNA sequences, including the $5^{\prime}$ end of $18 \mathrm{~S}$, the entire ITS1, 5.8S and ITS2 sequences, and the $3^{\prime}$ end of $28 \mathrm{~S}$ rDNA (Supplementary Table S2) from 44 specimens (indicated in yellow in supplementary

Table S1). Depending on the tick species, the size of the rDNA amplicon obtained varies from 4270 bp to $5054 \mathrm{bp}$. PCR products were purified using the kit Cleanpcr (CleanNA,

Waddinxveen, The Netherlands), fragmented to a size of $300 \mathrm{bp}$ and further used to construct libraries with the kit Nextera XT (Illumina, San Diego, California, USA). These libraries were then pooled together, indexed and pair-end sequenced on a Miseq (Illumina) sequencer using

174 a flow cell equipped with a V3, 600-cycle reagent cartridge.

We obtained $>24$ million reads after quality filtering and removing adaptors using the 
2010; M. Martin, 2011). Paired-end reads were de novo assembled using metaSPAdes v3.11 with k-mer sizes of 21, 33, 55, and 73 bp (Nurk, Meleshko, Korobeynikov, \& Pevzner, 2017). The resulting metaSPAdes contigs were binned into tick nuclear DNA and non-tick nuclear DNA groups through megablast in the GenBank nucleotide collection (Boratyn et al., 2013). These contigs were scaffolded, when applicable, by aligning to the complete sequences of the rRNA genes operon of A. americanum (GenBank AF291874), A. hebraeum (GenBank KY457489) and the partial sequence of A. marmoreum (GenBank KY457492) using MEGA (Kumar, Stecher, \& Tamura, 2016).

\section{Bacterial metabarcoding}

A 251-bp portion of the V4 variable region of the bacterial 16S rRNA gene was amplified individually for each DNA sample using a Multiplex PCR Kit (Qiagen) and universal primers (16SV4F: 5'-GTGCCAGCMGCCGCGGTAA-3' and 16SV4R: 5'-

GGACTACHVGGGTWTCTAATCC-3') (Galan et al., 2016). Amplified bacterial 16S rDNA products were purified and sequenced using an Illumina MiSeq platform (GenSeq, Montpellier University) and 251-bp end sequence reads were obtained,. All bioinformatic analyses were conducted using the pipeline Frogs (https://github.com/geraldinepascal/FROGS) (Escudié et al., 2018) as previously described (Binetruy et al., 2019)._One step of post-process operational taxonomic unit (OTU) affiliation was additionally performed through the Frogs pipeline on a Galaxy workbench (Escudié et al., 2018; Goecks et al., 2010). This step consists in aggregating OTUs that share 97\% of identity within $99 \%$ of the amplicon length, and it reduced the probability of keeping artefactual OTUs by resolving multi-hit ambiguities. To control the contamination during these procedure negative controls were performed (three negative extraction controls were included in all extraction series, two negative PCR controls were included in all PCR series). 
Moreover. OTUs having a maximal abundance in negative controls were discarded and falsepositive OTUs were removed by filtering OTU representing less than $0.005 \%$ of the OTU total abundance (Bokulich et al., 2013). Following this procedure, the microbiome was determined individually for almost all Amblyomma specimens $(n=142 / 143)$; however, the amount of DNA for the single specimen of A. sculptum was not sufficient.

A phylogenetic tree using OTU sequences and beta-diversity matrices based on this tree was assessed with FastTree and GUnifrac packages in R (J. Chen et al., 2012; Price, Dehal, \& Arkin, 2009), using the computational procedure described in (Binetruy, Dupraz, et al., 2019). Multidimensional scaling (MDS) plots were then generated using the package ggplot2 in R (Wickham, 2016). Permutational multivariate analysis of variance (PERMANOVA) implemented in the vegan package in R or pairwise PERMANOVA (Arbizu, 2017/2018) was further performed on the generalized UniFrac $(\alpha=0.5)$ dissimilarity matrix to evaluate the potential impact of the presence of symbionts and co-infection on bacterial diversity. The $P$ values of the pairwise PERMANOVA were corrected for multiple comparisons using Holm's method (Holm, 1979). A Mantel test was used to examine the association between microbial diversity (GUnifrac distance) and Amblyomma phylogeny using the ecodist package in R ( $n=9999$ permutations) (Goslee \& Urban, 2007).

\section{Multilocus typing of Coxiella-LE and Francisella-LE}

Coxiella-LE were genotyped through nested or semi-nested PCR amplification and sequencing of three housekeeping genes (16S rRNA, rpoB and groEL) previously developed for the Coxiella MLST methodology (Duron et al., 2015) (Supplementary Table S2). No Francisella-LE MLST was previously developed and we thus used the published genome of Francisella-LE F-Om strain (isolated from the soft tick Ornithodoros moubata [32]; 
for five genes (16S rRNA, rpoB, groEL, ftsZ and gyrB; Supplementary Table S2). Positive PCR products were purified and sequenced in both directions by Eurofins (Ebersberg, Germany). Sequence chromatograms were manually cleaned with Chromas Lite (http://www.technelysium.com.au/chromas_lite.html) and aligned with CLUSTALW implemented in MEGA 7 (Kumar et al., 2016; Thompson, Gibson, \& Higgins, 2002).

\section{Phylogenetic analyses}

Phylogenetic relationships were assessed using Amblyomma, Coxiella-LE and Francisella-LE sequences produced in this study and additional sequences available in GenBank (including Amblyomma, Coxiella-LE and Francisella-LE relatives and outgroups). GBLOCKS

(Castresana, 2000) was used to remove poorly aligned positions and to obtain unambiguous sequence alignments. All sequence alignments were also checked for putative recombinant regions using the RDP3 analysis package (D. P. Martin et al., 2010). The best fitting evolutionary models were determined using the Akaike information criterion and Bayesian information criterion with MEGA 7 (Kumar et al., 2016). Phylogenetic analyses were based on Bayesian inferences (BI) with MrBayes v3.2.7 (Ronquist et al., 2012). Two replicate analyses were run for 1 million generations. For each replicate, we ran one cold chain and three hot chains of the Markov chain Monte Carlo method, using a random starting tree and sampling trees every 100 generations and discarding $25 \%$ as burn-in. The remaining trees were used to calculate $50 \%$ majority-rule consensus trees.

The BI phylogenies of Amblyomma, Coxiella-LE and Francisella-LE were then used to conduct co-phylogenetic analyses using the Procrustean Approach to Cophylogeny (PACo) package in R (Balbuena, Míguez-Lozano, \& Blasco-Costa, 2013; Hutchinson, Cagua, Balbuena, Stouffer, \& Poisot, 2017). The significance of the co-phylogenetic tests was 
established by 100,000 random permutations of the two-association matrix. To test an effect of Rickettsia co-infection on the evolution of Francisella-LE and Coxiella-LE, linear regressions were computed in $\mathrm{R}$ using the matrix of genetic distances of these symbionts and the presence/absence of Rickettsia as an explicative variable.

\section{Ethics statement}

The use of the genetic resources was declared to the French Ministry of the Environment (reference TREL19028117S/156) and to Gabon government (entry authorization \#AE16008/PR/ANPN/SE/CS/AEPN and \#research authorization \#AR0013/16/MESRS/CENAREST/CG/CST/CSAR).

\section{Results}

Phylogeny of Amblyomma ticks

We first reconstructed the phylogenetic relationships among the 25 Amblyomma species using BI analyses based on large fragments of nuclear rDNA sequences obtained from one-to-two specimens per species (Figure 1). The findings support a monophyletic origin of the five African Amblyomma species (AF group): They cluster together in a robust clade nested among the 20 New World Amblyomma species (NW group), suggesting an American origin of these African species (Figure 1). Moreover, the phylogeny of Amblyomma also parallels the tick host-range at least in the NW group: Closely related Amblyomma species often share the same host species, such as $A$. dissimile and A. rotundatum feeding on poikilotherms (reptiles and amphibians) or A. latepunctatum, A. scalpturatum and A. naponense feeding mainly on tapirs and Suidae (Figure 1). However, in other cases, closely related Amblyomma species use different host species, such as A. ovale, a generalist species feeding on a diversity 
of domestic and wild animals, and A. varium, a specialized species feeding on arboreal vertebrates.

\section{Microbial diversity and symbiont prevalence}

We further examined the whole bacterial diversity of 24 out of the 25 Amblyomma species ( $n=142$ specimens, one-to-ten specimens were examined per species) via high-throughput $16 \mathrm{~S}$ rDNA sequencing. The amount of DNA for the $25^{\text {th }}$ Amblyomma species, A. sculptum $(n=1)$, was not sufficient to perform bacterial barcoding (this specimen was already PCR-typed for Coxiella-LE and Francisella-LE, as further detailed). After filtration of false-positive OTUs and contaminants (Binetruy, Dupraz, et al., 2019; Birer, Tysklind, Zinger, \& Duplais, 2017), 4,364,360 reads distributed in 195 OTUs were obtained (Table S3). Coxiella-LE and Francisella-LE were the most abundant bacterial genera representing $33.6 \%$ and $33.1 \%$ of the total number of reads, respectively (Figure 2 and Supplementary Table S1). Other bacterial genera were found: In most cases, each represented a negligible part of the $16 \mathrm{~S}$ rDNA reads when present. A remarkable exception to this pattern was the presence of abundant intracellular bacteria belonging to the Rickettsia genus in 16 Amblyomma species (Figure 2 and Supplementary Table S1). Other exceptions included the A. romitii samples for which no Coxiella-LE and Francisella-LE reads were detected: Most reads were assigned to a tickborne pathogen, Ehrlichia sp. In A. loculosum and Amblyomma sp. samples, Coxiella-LE reads were detected but reads of other bacteria were more abundant. Since the A. loculosum and Amblyomma sp. samples were not bleach-treated prior to DNA extraction (as done for the other samples), the abundant presence of these bacteria may be due to the cuticular bacteriome, as recently observed in another Amblyomma species (Binetruy, Dupraz, et al., 2019). 
The structure of microbial diversity was not globally impacted by the phylogenetic proximity among Amblyomma species: The dendrogram of microbial diversity did not parallel the Amblyomma phylogeny (Mantel two-tailed test, $R=0.16, P=0.19$ ) (Supplementary Figure $\mathrm{S} 1$ ). However, the MDS plot suggested an effect of tick species on bacterial diversity (Supplementary Figure S2), as confirmed by the PERMANOVA analysis $\left(R^{2}=0.51\right.$, $P=0.001)$. Further testing showed a clear separation between Coxiella-LE-infected tick species/specimens and Francisella-LE-infected ones (pairwise PERMANOVA analysis, $R^{2}=0.31$, adjusted $P$ for multiple comparisons=0.0003; Figure $\left.3 \mathrm{~A}\right)$. Rickettsia also structures the bacterial diversity but to a lesser extent than Coxiella-LE and Francisella-LE (PERMANOVA, $R^{2}=0.11, P=0.001$; Figure 3B). The Coxiella LE and Francisella-LE clusters are actually structured into two sub-clusters each, fitting with the presence of Rickettsia (Figure 3c) as corroborated by PERMANOVA analyses: (1) Coxiella-LE without Rickettsia vs. Coxiella-LE with Rickettsia $\left(R^{2}=0.2\right.$, adjusted $P$ for multiple comparisons=0.0015), and (2) Francisella -LE without Rickettsia vs. Francisella -LE with Rickettsia $\left(R^{2}=0.22\right.$, adjusted $P$ for multiple comparisons=0.0015).

Of the 25 Amblyomma species (including A. sculptum) examined for the presence of CoxiellaLE and Francisella-LE, 24 were infected by one or both of these symbionts: 11 Amblyomma species harbour only Coxiella-LE, 13 species only Francisella-LE and three species both (Figure 2, Supplementary Table S1). Only A. romitii was not infected by Coxiella-LE and Francisella-LE but this may be explained by the presence of the tick-borne pathogen Ehrlichia sp., which may mask the presence of other bacteria. In the 24 infected Amblyomma species, Coxiella-LE and Francisella-LE were not randomly associated (Fisher's exact test, $P=0.001)$ : These two symbionts co-occurred in the same tick species less frequently than expected by chance (exclusion pattern), meaning that their distribution across tick species was 
strongly dependent on each other. Conversely, neither Coxiella-LE nor Francisella-LE showed a non-random association with Rickettsia (Fisher's exact tests, $P=0.99$ and 0.68 , respectively). However, Coxiella-LE and Francisella-LE had a patchy and quite uniform distribution along the Amblyomma phylogeny: While some closely related Amblyomma species were infected by the same symbiont genus (e.g. A. dissimile and A. rotundatum by Francisella-LE, or A. latepunctatum, A. scalpturatum and A. naponense by Coxiella-LE), others were not (e.g. A. americanum, infected by Coxiella-LE, and A. oblongoguttatum by Francisella-LE) (Figure 2). This distribution pattern paralleled partly the tick host-range: Indeed, $A$. dissimile and $A$. rotundatum that are related and specialized for poikilotherms were both infected by Francisella-LE (Figure 1, 2).

In the tick species they infect, Coxiella-LE and Francisella-LE were present in most specimens examined (Figure 2, Supplementary Table S1). However, a more contrasted pattern was apparent in the three Amblyomma species that were co-infected by Coxiella-LE and Francisella-LE: (i) in A. geayi, of the 10 examined specimens, seven were infected by Coxiella-LE in one locality, but in other localities, two specimens were infected by Francisella-LE and one was co-infected by Coxiella-LE and Francisella-LE; (ii) in A. latepunctatum, of the four examined specimens, three specimens of the same locality were coinfected by Coxiella-LE and Francisella-LE but one specimen from another locality was only infected by Coxiella-LE; (iii) in A. sculptum, the single examined specimen was co-infected by Coxiella-LE and Francisella-LE (Figure 2, Supplementary Table S1). In contrast to Coxiella-LE and Francisella-LE, the prevalence of Rickettsia was heterogeneous, with infection frequencies ranging from $14 \%$ to $100 \%$ depending on tick species (Figure 2, Supplementary Table S1). In all cases, Rickettsia was found with either Coxiella-LE (seven 
Amblyomma species) or Francisella-LE (seven species) or both (two species). Neither

Rickettsia nor Coxiella-LE nor Francisella-LE shows infection-biased sex ratio: within each Amblyomma species, the prevalence of infection did not differ between males and females (Fisher's exact test, all $P>0.1$ ).

Evolutionary history of Coxiella-LE and Francisella-LE symbioses

Sequencing of three Coxiella MLST genes (16S rRNA, rpoB and groEL) led to the identification of 14 genetically different Coxiella-LE in a subset of 32 specimens representing the 14 infected Amblyomma species (one to four specimens per species were examined). Each Amblyomma species was infected by a genetically distinct Coxiella-LE and no variation of Coxiella-LE was observed among specimens belonging to the same Amblyomma species. We observed no sign of recombination in the Coxiella-LE data set (all $P>0.05$ for the GENECONV and RDP recombination-detection tests) and we thus used the $16 \mathrm{~S}$ rRNA, rpoB and groEL concatenated sequences for BI analyses. Comparisons with other sequences available on GenBank showed that the Coxiella-LE of Amblyomma are polyphyletic: They were scattered into different well-supported clusters among Coxiella-LE of other tick species (Supplementary Figure S3). Indeed, the Coxiella-LE of A. variegatum, A. tholloni and A. splendidum form a monophyletic clade that is more closely related to the Coxiella-LE of Ixodes tick species than to the Coxiella-LE of other Amblyomma species. Similarly, the Coxiella-LE clade of A. cajennense, A. sculptum and A. americanum is more closely related to the Coxiella-LE of Dermacentor tick species. This pattern is suggestive of recurrent HT events of Coxiella-LE among tick species. However, the Coxiella-LE clusters of Amblyomma species can be gathered into two main groups, one with all the Coxiella-LE of NW Amblyomma species and the other with all the Coxiella-LE of AF Amblyomma species (Supplementary Figure S3). This pattern suggests an effect of phylogeographic drivers in 
structuring the evolution of Coxiella-LE. This is strongly supported by the co-phylogeny analysis between Coxiella-LE and Amblyomma phylogenies: There is a significant topological congruence between their phylogeny (PACo analysis, $P=0.0001$; Figure $4 \mathrm{~A}$ ). This shows that co-cladogenesis with Coxiella-LE occurred during the radiation of Amblyomma.

Sequencing of five Francisella MLST genes (16S rRNA, rpoB, groEL, ftsZ and gyrB) led to the identification of 15 genetically different Francisella-LE in a subset of 28 tick specimens representing the 13 infected Amblyomma species (one to four specimens per species were used). There was only one Francisella-LE in each Amblyomma species, except A. pacae and A. rotundatum in which two and three genetically distinct Francisella-LE, respectively, were present in specimens from different localities. Each Amblyomma species harbours genetically distinct Francisella-LE, except for A. geayi and A. latepunctatum that harbour identical Francisella-LE on the basis of their MSLT sequences (Supplementary Figure S4). Owing to the lack of Francisella-LE rpoB, groEL, ftsZ and $g y r B$ gene sequences available in GenBank before this study (with the exception of two published Francisella-LE genomes), the BI phylogenetic analysis between the Francisella-LE of Amblyomma and those of other tick species (with sequences available in GenBank) was made using only their 16S rRNA nucleotidic sequences (Supplementary Figure S5). No Francisella-LE subclade specific to Amblyomma exists along the 16S rRNA phylogenetic tree: The Francisella-LE of Amblyomma are instead scattered among Francisella-LE of other tick genera suggesting recurrent HT events among unrelated tick species (Supplementary Figure S5). However, the inner topology of the Francisella-LE clade based on 16S rRNA gene sequences remained too poorly resolved in many cases (as shown by low support values of inner branches) to infer the exact relatedness among all Francisella-LE. We thus further reconstructed the phylogenetic relationships between Francisella-LE using BI analyses based on their 16S rRNA, rpoB, 
groEL, fts $Z$ and gyrB nucleotidic sequences. We observed no sign of recombination in the Francisella-LE data set (all $P>0.05$ for the GENECONV and RDP recombination-detection tests) and we thus used the $16 \mathrm{~S}$ rRNA, rpoB, groEL, ftsZ and gyrB concatenated sequences for analyses. Conversely to Coxiella-LE, there was no apparent co-cladogenesis or phylogeographic pattern along the Francisella-LE phylogeny (Supplementary Figure S4). Indeed, the Francisella-LE of A. sculptum (NW group) is closely related to the FrancisellaLE of an unrelated Amblyomma species, A. paulopunctatum (AF group). In addition, the Francisella-LE of A. sculptum and A. paulopunctatum are more closely related to the Francisella-LE of the soft tick O. moubata than to the Francisella-LE of other Amblyomma species. The co-phylogeny analysis also showed no significant signal of congruence between the Francisella-LE and Amblyomma phylogenies (PACo analysis, $P=0.06$ ): Only HT events seem to have impacted the distribution Francisella-LE across Amblyomma phylogeny (Figure 4B). Interestingly, while co-infection with Rickettsia along the Coxiella-LE phylogeny is random (linear model, adjusted $R^{2}=-0.01$, F-stat $=0.60, P=0.56$; Supplementary Figure S6A), it is not so along the Francisella-LE phylogeny (adjusted $R^{2}=0.15$, F-stat=6.90, $P=0.002$; Figure S6B): Co-infections with Rickettsia are more common with certain Francisella-LE subclades than with others.

\section{Discussion}

Three intracellular bacterial genera, Coxiella-LE, Francisella-LE and Rickettsia, are widespread across the 25 species of Amblyomma ticks we examined in this study. Only a few other bacteria have been detected and none is so uniformly present in Amblyomma. However, the structure of the microbiomes does not mirror the Amblyomma phylogeny and closely related Amblyomma commonly harbour divergent microbiomes. As expected, almost all Amblyomma species were found to harbour a nutritive obligate symbiont, Coxiella-LE or 
Francisella-LE, both able to synthesize B vitamins but with a clear exclusion pattern between them: Coxiella-LE was found as a single infection in 11 Amblyomma species, Francisella-LE in 13 species and co-infection was seen in only three species. Despite the co-evolved and obligate interactions of ticks with their mutualistic partners, we detected evidence of symbiont replacements during radiation of Amblyomma, raising questions regarding the ecological and evolutionary factors underlying replacements.

The comparison of symbiont and tick phylogenies revealed that the Coxiella-LE symbiosis is ancient and arose in the early evolution of the Amblyomma genus. Hence, some Coxiella-LE are specialized for their Amblyomma hosts, with an ancient acquisition followed by codiversification, meaning that the persistence of Coxiella-LE through vertical transmission is stable over the duration of Amblyomma species diversification. A very similar codiversification pattern has also been reported for Coxiella-LE symbiosis in the Rhipicephalus tick genus (Duron et al., 2017). However, the spread of Coxiella-LE was more complex in Amblyomma: The infections found in some Amblyomma species are distantly related and do not form an Amblyomma-specific clade. Rather, phylogenetics shows that Coxiella-LE of Amblyomma are actually scattered among Coxiella-LE of other tick genera such as Ixodes and Dermacentor. Only extensive HT of Coxiella-LE among tick genera may explain these phylogenetic incongruences. Since facultative, but not obligate, symbionts can undergo HT between host species (Bennett \& Moran, 2015; McCutcheon et al., 2019; Nancy A. Moran et al., 2008; Wernegreen, 2012), this suggests that some Coxiella-LE are facultative symbionts of ticks. Interestingly, Coxiella-LE is a facultative symbiont in some Ixodes species, such as $I$. ricinus and I. uriae (Duron et al., 2017; Duron, Jourdain, \& McCoy, 2014; Duron et al., 2015). The phylogenetic proximity of Coxiella-LE of Ixodes spp. with the Coxiella-LE of A. variegatum, A. splendidum and A. tholloni thus suggests that a facultative Coxiella-LE of 
Ixodes spp. had an early jump to the Amblyomma ancestor of these species before replacing the ancestral obligate symbiont and evolving obligate nutritional symbioses with current species.

The infection dynamics of Francisella-LE is different to that of Coxiella-LE. While the Francisella-LE of ticks form a monophyletic clade within the Francisella genus (Duron et al., 2017, 2018), we observed frequent HT events between unrelated tick species. No cocladogenesis signal with Amblyomma is apparent along the phylogenies, meaning that current Francisella-LE arose only recently in this genus. The presence of unrelated Francisella-LE in Amblyomma further indicates that several independent acquisitions of Francisella-LE have occurred during the radiation of this tick genus. These acquisitions have likely come at the expense of the Coxiella-LE with their ultimate replacement by Francisella-LE. The AF Amblyomma group is illustrative of this process: Most species (A. splendidum, A. variegatum, A. tholloni and A. loculosum) are infected by Coxiella-LE with a strong co-cladogenesis pattern, but one species (A. paulopunctatum) is infected by Francisella-LE. This pattern suggests that Francisella-LE has replaced the Coxiella-LE primarily present in the A. paulopunctatum ancestor. Other examples include the monophyletic group formed by $A$. americanum, A. oblongoguttatum, A. cajennense and A. sculptum: Coxiella-LE has codiverged with all species but one, since here Francisella-LE eliminated the Coxiella-LE primarily present in the A. oblongoguttatum ancestor. In addition, A. sculptum is co-infected at the individual level by an ancestral Coxiella-LE (i.e. showing a co-cladogenesis pattern with Coxiella-LE of the A. sculptum relatives) and a recently acquired Francisella-LE. This pattern suggests that HT of Francisella-LE within Amblyomma communities is recent, likely ongoing, and that co-infections with ancestral Coxiella-LE are only transitory. The preferential association of some Francisella-LE with Rickettsia further implies that these co- 
infections may be important drivers since some Rickettsia are also able to synthesize folate (B9 vitamin) (Hunter et al., 2015) and thus to participate in nutritional symbiosis along with Francisella-LE. Under this hypothesis, Francisella-LE and Rickettsia are cooperating together, and each may fulfil essential metabolic functions not ensured by the others. They may also act together to replace ancestral Coxiella-LE.

Some biological traits of these symbioses are indicative of how and why Francisella-LE and some Coxiella-LE are both eliminating ancestral Coxiella-LE in Amblyomma ticks.

Francisella-LE or Coxiella-LE were occasionally detected in the salivary glands of several tick species (Budachetri et al., 2014; Buysse et al., 2019; Klyachko, Stein, Grindle, Clay, \& Fuqua, 2007) suggesting that ticks may inject part of their symbionts during feeding. Ticks, unlike other arthropod vectors, often attach and aggregate on the host for several days to obtain a meal, a process termed 'co-feeding'. The spatiotemporal proximity of ticks during co-feeding may favour the HT of Francisella-LE and Coxiella-LE between conspecifics but also between different tick species, as commonly observed for tick-borne pathogens (Voordouw, 2015; Wright, Sonenshine, Gaff, \& Hynes, 2015). This process may lead to local or systemic infections in vertebrates since a few cases of opportunistic Coxiella-LE infections have been reported after tick feeding (Shivaprasad et al., 2008; Vapniarsky, Barr, \& Murphy, 2012; Woc-Colburn et al., 2008, p.). This mode of transmission may be particularly significant for Francisella-LE and Coxiella-LE by leading to co-infections with ancestral Coxiella-LE in ticks. Interestingly, we found two Amblyomma species, A. geayi and A. latepunctatum, that are co-infected by their respective ancestral Coxiella-LE but that also share the same Francisella-LE, which is also closely related to the Francisella-LE of $A$. dissimile. The genetic proximity of Francisella-LE in these three unrelated Amblyomma species is suggestive of recent HT events through co-feeding: While these three Amblyomma 
species feed on very different vertebrate hosts, hampering the possibility of co-feeding, the immature stages of $A$. dissimile are commonly found in diverse mammals or birds (Binetruy, Chevillon, de Thoisy, Garnier, \& Duron, 2019; Guglielmone \& Nava, 2010; Scott \& Durden, 2015), suggesting that they may be ecological bridges driving HT of Francisella-LE across tick species.

By replacing the ancestral Coxiella-LE, the novel symbiont colonizes a pre-adapted tick physiological environment that requires the provision of B vitamin: Thus, it must be able to synthesize these compounds as the ancestral Coxiella-LE did. The presence of several B vitamin biosynthesis pathways is ancestral in the Coxiella and Francisella genera, and all members of these genera, including pathogenic species that are also all intracellular, have conserved these abilities through their radiation (Duron et al., 2018; Gerhart et al., 2016; Meibom \& Charbit, 2010; Rowe \& Huntley, 2015; Smith et al., 2015; van Schaik, Chen, Mertens, Weber, \& Samuel, 2013): All Francisella-LE and Coxiella-LE are already preadapted to nutritional symbioses with ticks. Beyond B vitamins, the replacement of ancestral Coxiella-LE suggests that the new symbiont could supply an additional benefit that the ancestral Coxiella-LE was unable to supply to ticks, thereby out-competing them. The genomes of ancient beneficial endosymbionts have lost most of their gene contents from their ancestor, being usually small in size and dense in gene content but also suffering Muller's ratchet, with fixation of deleterious mutations through genetic drift (McCutcheon et al., 2019; McCutcheon \& Moran, 2012; N. A. Moran, 1996; Rispe \& Moran, 2000). This evolution towards massive genomic reduction is obvious for the Coxiella-LE of A. americanum, which have a genome of only 0.66 Mb (Smith et al., 2015), but not for the Francisella-LE of $A$. maculatum: Its genome is $1.56 \mathrm{Mb}$, and although half is pseudogenized, it may have a higher biosynthetic capability (Gerhart et al., 2016). Similar variation is also reported between 
Coxiella-LE with some genomes reaching ca. 1.5 Mb (Gottlieb et al., 2015; Ramaiah \&

Dasch, 2018), suggesting that some Coxiella-LE have greater biosynthetic capabilities than

others. However, other processes may act on the Coxiella-LE replacement. Indeed, ancestral

Coxiella-LE may have evolved too reduced (degraded) genomes and become maladapted,

opening the road to replacement by a new symbiont. This degeneration-replacement model has been proposed for other arthropods such as cicadas (Campbell et al., 2015; Lukasik et al., 2018; Matsuura et al., 2018), but replacements are expected to be transient making them difficult to observe (McCutcheon et al., 2019). In Amblyomma, the observations of three species with co-infections by ancestral Coxiella-LE and recently acquired Francisella-LE may correspond to this transient state before extinction of Coxiella-LE.

That ecological specialization to strict haematophagy is driven by nutritional symbiotic interactions is beyond doubt for ticks. The present study, nevertheless, shows that nutritional symbiosis in the Amblyomma genus is not stable state, being impacted by competition between Coxiella-LE and Francisella-LE or between Coxiella-LE themselves. We potentialy underestimate the amplitude of this dynamics: our intraspecific sampling was low for some tick species and this may have led to an underestimation of the Francisella-LE frequency. Indeed, in the cases of low prevalence, most samples will be found not infected by Francisella-LE while other members of the species, not sampled and tested, are in fact infected. Precisely, this pattern is found in this study: we found variation Francisella-LE infection pattern between sampling localities in three tick species in which just few few specimens were infected, which clearly indicates this potential for false negatives arising from insufficient sampling. Anyway, ticks now march on with their recently acquired Francisella- 
competition between symbionts with similar metabolic capabilities and the differential degree of genome reduction.

\section{References}

Ahantarig, A., Trinachartvanit, W., Baimai, V., \& Grubhoffer, L. (2013). Hard ticks and their bacterial endosymbionts (or would be pathogens). Folia Microbiologica, 58(5), 419-428. doi: $10.1007 / \mathrm{s} 12223-013-0222-1$

Arbizu, P. M. (2018). Pairwise multilevel comparison using adonis. Contribute to pmartinezarbizu/pairwiseAdonis development by creating an account on GitHub [R]. Retrieved from https://github.com/pmartinezarbizu/pairwiseAdonis (Original work published 2017)

Balbuena, J. A., Míguez-Lozano, R., \& Blasco-Costa, I. (2013). PACo: A Novel Procrustes Application to Cophylogenetic Analysis. PLOS ONE, 8(4), e61048. doi: 10.1371/journal.pone.0061048

Bennett, G. M., \& Moran, N. A. (2015). Heritable symbiosis: The advantages and perils of an evolutionary rabbit hole. Proceedings of the National Academy of Sciences of the United States of America, 112(33), 10169-10176. doi: 10.1073/pnas.1421388112

Binetruy, F., Chevillon, C., de Thoisy, B., Garnier, S., \& Duron, O. (2019). Survey of ticks in French Guiana. Ticks and Tick-Borne Diseases, 10(1), 77-85. doi: 10.1016/j.ttbdis.2018.09.003

Binetruy, F., Dupraz, M., Buysse, M., \& Duron, O. (2019). Surface sterilization methods impact measures of internal microbial diversity in ticks. Parasites \& Vectors, 12(1), 268. doi: $10.1186 / \mathrm{s} 13071-019-3517-5$

Birer, C., Tysklind, N., Zinger, L., \& Duplais, C. (2017). Comparative analysis of DNA extraction methods to study the body surface microbiota of insects: A case study with ant cuticular bacteria. Molecular Ecology Resources, 17(6), e34-e45. doi: 10.1111/1755-0998.12688 
Bokulich, N. A., Subramanian, S., Faith, J. J., Gevers, D., Gordon, J. I., Knight, R., ... Caporaso, J. G. (2013). Quality-filtering vastly improves diversity estimates from Illumina amplicon sequencing. Nature Methods, 10(1), 57-59. doi: 10.1038/nmeth.2276

Bonnet, S. I., Binetruy, F., Hernández-Jarguín, A. M., \& Duron, O. (2017). The Tick Microbiome: Why Non-pathogenic Microorganisms Matter in Tick Biology and Pathogen Transmission. Frontiers in Cellular and Infection Microbiology, 7. doi: 10.3389/fcimb.2017.00236

Boratyn, G. M., Camacho, C., Cooper, P. S., Coulouris, G., Fong, A., Ma, N., ... Zaretskaya, I. (2013). BLAST: a more efficient report with usability improvements. Nucleic Acids Research, 41(Web Server issue), W29-W33. doi: 10.1093/nar/gkt282

Budachetri, K., Browning, R. E., Adamson, S. W., Dowd, S. E., Chao, C.-C., Ching, W.-M., \& Karim, S. (2014). An insight into the microbiome of the Amblyomma maculatum (Acari: Ixodidae). Journal of Medical Entomology, 51(1), 119-129. doi: 10.1603/me12223

Buysse, M., Plantard, O., McCoy, K. D., Duron, O., \& Menard, C. (2019). Tissue localization of Coxiellalike endosymbionts in three European tick species through fluorescence in situ hybridization. Ticks and Tick-Borne Diseases, 10(4), 798-804. doi: 10.1016/j.ttbdis.2019.03.014

Campbell, M. A., Leuven, J. T. V., Meister, R. C., Carey, K. M., Simon, C., \& McCutcheon, J. P. (2015). Genome expansion via lineage splitting and genome reduction in the cicada endosymbiont Hodgkinia. Proceedings of the National Academy of Sciences, 112(33), 10192-10199. doi: $10.1073 /$ pnas. 1421386112

Castresana, J. (2000). Selection of Conserved Blocks from Multiple Alignments for Their Use in Phylogenetic Analysis. Molecular Biology and Evolution, 17(4), 540-552. doi: 10.1093/oxfordjournals.molbev.a026334

Chen, J., Bittinger, K., Charlson, E. S., Hoffmann, C., Lewis, J., Wu, G. D., ... Li, H. (2012). Associating microbiome composition with environmental covariates using generalized UniFrac distances. Bioinformatics (Oxford, England), 28(16), 2106-2113. doi: 10.1093/bioinformatics/bts342 
Chen, X., Li, S., \& Aksoy, S. (1999). Concordant evolution of a symbiont with its host insect species: molecular phylogeny of genus Glossina and its bacteriome-associated endosymbiont, Wigglesworthia glossinidia. Journal of Molecular Evolution, 48(1), 49-58.

Childs, J. E., \& Paddock, C. D. (2003). The ascendancy of Amblyomma americanum as a vector of pathogens affecting humans in the United States. Annual Review of Entomology, 48, 307337. doi: $10.1146 /$ annurev.ento.48.091801.112728

Clay, K., Klyachko, O., Grindle, N., Civitello, D., Oleske, D., \& Fuqua, C. (2008). Microbial communities and interactions in the lone star tick, Amblyomma americanum. Molecular Ecology, 17(19), 4371-4381.

Duron, O., Binetruy, F., Noël, V., Cremaschi, J., McCoy, K. D., Arnathau, C., ... Chevillon, C. (2017). Evolutionary changes in symbiont community structure in ticks. Molecular Ecology, 26(11), 2905-2921. doi: 10.1111/mec.14094

Duron, O., Jourdain, E., \& McCoy, K. D. (2014). Diversity and global distribution of the Coxiella intracellular bacterium in seabird ticks. Ticks and Tick-Borne Diseases, 5(5), 557-563. doi: 10.1016/j.ttbdis.2014.04.003

Duron, O., Morel, O., Noël, V., Buysse, M., Binetruy, F., Lancelot, R., ... Vial, L. (2018). Tick-Bacteria Mutualism Depends on B Vitamin Synthesis Pathways. Current Biology, 28(12), 18961902.e5. doi: 10.1016/j.cub.2018.04.038

Duron, O., Noël, V., McCoy, K. D., Bonazzi, M., Sidi-Boumedine, K., Morel, O., ... Chevillon, C. (2015). The Recent Evolution of a Maternally-Inherited Endosymbiont of Ticks Led to the Emergence of the Q Fever Pathogen, Coxiella burnetii. PLoS Pathogens, 11(5), e1004892. doi: 10.1371/journal.ppat.1004892

Duron, O., Wilkes, T. E., \& Hurst, G. D. D. (2010). Interspecific transmission of a male-killing bacterium on an ecological timescale. Ecology Letters, 13(9), 1139-1148. doi: 10.1111/j.14610248.2010.01502.x 
Engelstädter, J., \& Hurst, G. D. D. (2009). The Ecology and Evolution of Microbes that Manipulate Host Reproduction. Annual Review of Ecology, Evolution, and Systematics, 40(1), 127-149. doi: 10.1146/annurev.ecolsys.110308.120206

Escudié, F., Auer, L., Bernard, M., Mariadassou, M., Cauquil, L., Vidal, K., ... Pascal, G. (2018). FROGS: Find, Rapidly, OTUs with Galaxy Solution. Bioinformatics (Oxford, England), 34(8), 12871294. doi: 10.1093/bioinformatics/btx791

Falony, G., Joossens, M., Vieira-Silva, S., Wang, J., Darzi, Y., Faust, K., ... Raes, J. (2016). Populationlevel analysis of gut microbiome variation. Science (New York, N.Y.), 352(6285), 560-564. doi: 10.1126/science.aad3503

Ferrari, J., \& Vavre, F. (2011). Bacterial symbionts in insects or the story of communities affecting communities. Philosophical Transactions of the Royal Society of London. Series B, Biological Sciences, 366(1569), 1389-1400. doi: 10.1098/rstb.2010.0226

Galan, M., Razzauti, M., Bard, E., Bernard, M., Brouat, C., Charbonnel, N., ... Cosson, J.-F. (2016). 16 S rRNA Amplicon Sequencing for Epidemiological Surveys of Bacteria in Wildlife. MSystems, 1(4). doi: $10.1128 / \mathrm{mSystems.00032-16}$

Gerhart, J. G., Moses, A. S., \& Raghavan, R. (2016). A Francisella-like endosymbiont in the Gulf Coast tick evolved from a mammalian pathogen. Scientific Reports, 6, 33670. doi: $10.1038 /$ srep33670

Goecks, J., Nekrutenko, A., Taylor, J., \& The Galaxy Team. (2010). Galaxy: a comprehensive approach for supporting accessible, reproducible, and transparent computational research in the life sciences. Genome Biology, 11(8), R86. doi: 10.1186/gb-2010-11-8-r86

Goslee, S. C., \& Urban, D. L. (2007). The ecodist Package for Dissimilarity-based Analysis of Ecological Data. Journal of Statistical Software, 22(1), 1-19. doi: 10.18637/jss.v022.i07

Gottlieb, Y., Ghanim, M., Gueguen, G., Kontsedalov, S., Vavre, F., Fleury, F., \& Zchori-Fein, E. (2008). Inherited intracellular ecosystem: symbiotic bacteria share bacteriocytes in whiteflies. FASEB 

Journal: Official Publication of the Federation of American Societies for Experimental Biology, 22(7), 2591-2599. doi: 10.1096/fj.07-101162

Gottlieb, Y., Lalzar, I., \& Klasson, L. (2015). Distinctive Genome Reduction Rates Revealed by Genomic Analyses of Two Coxiella-Like Endosymbionts in Ticks. Genome Biology and Evolution, 7(6), 1779-1796. doi: 10.1093/gbe/evv108

Gould, A. L., Zhang, V., Lamberti, L., Jones, E. W., Obadia, B., Korasidis, N., ... Ludington, W. B. (2018). Microbiome interactions shape host fitness. Proceedings of the National Academy of Sciences, 115(51), E11951-E11960. doi: 10.1073/pnas.1809349115

Groussin, M., Mazel, F., Sanders, J. G., Smillie, C. S., Lavergne, S., Thuiller, W., \& Alm, E. J. (2017). Unraveling the processes shaping mammalian gut microbiomes over evolutionary time. Nature Communications, 8, 14319. doi: 10.1038/ncomms14319

Guglielmone, A. A., Estrada-Peña, A., Keirans, J. E., \& Robbins, R. G. (2003). Ticks (Acari: Ixodida) of the Neotropical Zoogeographic Region. Universiteit Utrecht, J. Bovy-Verbeek (public relations ICTTD).

Guglielmone, A. A., \& Nava, S. (2010). Hosts of Amblyomma dissimile Koch, 1844 and Amblyomma rotundatum Koch, 1844 (Acari: Ixodidae). Zootaxa, 2541, 27-49.

Guizzo, M. G., Parizi, L. F., Nunes, R. D., Schama, R., Albano, R. M., Tirloni, L., ... Oliveira, P. L. (2017). A Coxiella mutualist symbiont is essential to the development of Rhipicephalus microplus. Scientific Reports, 7(1), 17554. doi: 10.1038/s41598-017-17309-x

Hanning, I., \& Diaz-Sanchez, S. (2015). The functionality of the gastrointestinal microbiome in nonhuman animals. Microbiome, 3. doi: 10.1186/s40168-015-0113-6

Holm, S. (1979). A Simple Sequentially Rejective Multiple Test Procedure. Scandinavian Journal of Statistics, 6(2), 65-70. Retrieved from JSTOR.

Hosokawa, T., Koga, R., Kikuchi, Y., Meng, X.-Y., \& Fukatsu, T. (2010). Wolbachia as a bacteriocyteassociated nutritional mutualist. Proceedings of the National Academy of Sciences of the United States of America, 107(2), 769-774. doi: 10.1073/pnas.0911476107 
Hunter, D. J., Torkelson, J. L., Bodnar, J., Mortazavi, B., Laurent, T., Deason, J., ... Zhong, J. (2015). The Rickettsia Endosymbiont of Ixodes pacificus Contains All the Genes of De Novo Folate Biosynthesis. PLOS ONE, 10(12). doi: 10.1371/journal.pone.0144552

Hutchinson, M. C., Cagua, E. F., Balbuena, J. A., Stouffer, D. B., \& Poisot, T. (2017). paco: implementing Procrustean Approach to Cophylogeny in R. Methods in Ecology and Evolution, 8(8), 932-940. doi: 10.1111/2041-210X.12736

Jaenike, J. (2012). Population genetics of beneficial heritable symbionts. Trends in Ecology \& Evolution, 27(4), 226-232. doi: 10.1016/j.tree.2011.10.005

Jousselin, E., Cœur d'Acier, A., Vanlerberghe-Masutti, F., \& Duron, O. (2013). Evolution and diversity of Arsenophonus endosymbionts in aphids. Molecular Ecology, 22(1), 260-270. doi: 10.1111/mec.12092

Jousselin, E., Desdevises, Y., \& Coeur d'acier, A. (2009). Fine-scale cospeciation between Brachycaudus and Buchnera aphidicola: bacterial genome helps define species and evolutionary relationships in aphids. Proceedings. Biological Sciences, 276(1654), 187-196. doi: $10.1098 / \mathrm{rspb} .2008 .0679$

Klyachko, O., Stein, B. D., Grindle, N., Clay, K., \& Fuqua, C. (2007). Localization and visualization of a coxiella-type symbiont within the lone star tick, Amblyomma americanum. Applied and Environmental Microbiology, 73(20), 6584-6594. doi: 10.1128/AEM.00537-07

Koga, R., \& Moran, N. A. (2014). Swapping symbionts in spittlebugs: evolutionary replacement of a reduced genome symbiont. The ISME Journal, 8(6), 1237-1246. doi: 10.1038/ismej.2013.235

Koga, R., Nikoh, N., Matsuura, Y., Meng, X.-Y., \& Fukatsu, T. (2013). Mealybugs with distinct endosymbiotic systems living on the same host plant. FEMS Microbiology Ecology, 83(1), 93100. doi: 10.1111/j.1574-6941.2012.01450.x

Kumar, S., Stecher, G., \& Tamura, K. (2016). MEGA7: Molecular Evolutionary Genetics Analysis Version 7.0 for Bigger Datasets. Molecular Biology and Evolution, 33(7), 1870-1874. doi: $10.1093 / \mathrm{molbev} / \mathrm{msw} 054$ 
Kurtti, T. J., Felsheim, R. F., Burkhardt, N. Y., Oliver, J. D., Heu, C. C., \& Munderloh, U. G. (2015). Rickettsia buchneri sp. nov., a rickettsial endosymbiont of the blacklegged tick Ixodes scapularis. International Journal of Systematic and Evolutionary Microbiology, 65(Pt 3), 965970. doi: $10.1099 /$ ijs.0.000047

Ley, R. E., Hamady, M., Lozupone, C., Turnbaugh, P., Ramey, R. R., Bircher, J. S., ... Gordon, J. I. (2008). Evolution of mammals and their gut microbes. Science (New York, N.Y.), 320(5883), 16471651. doi: $10.1126 /$ science. 1155725

Łukasik, P., Nazario, K., Leuven, J. T. V., Campbell, M. A., Meyer, M., Michalik, A., ... McCutcheon, J. P. (2018). Multiple origins of interdependent endosymbiotic complexes in a genus of cicadas. Proceedings of the National Academy of Sciences, 115(2), E226-E235. doi: 10.1073/pnas.1712321115

Margulis, L. (1993). Origins of species: acquired genomes and individuality. Biosystems, 31(2), 121125. doi: 10.1016/0303-2647(93)90039-F

Martin, D. P., Lemey, P., Lott, M., Moulton, V., Posada, D., \& Lefeuvre, P. (2010). RDP3: a flexible and fast computer program for analyzing recombination. Bioinformatics (Oxford, England), 26(19), 2462-2463. doi: 10.1093/bioinformatics/btq467

Martin, M. (2011). Cutadapt removes adapter sequences from high-throughput sequencing reads. EMBnet.Journal, 17(1), 10-12. doi: 10.14806/ej.17.1.200

Matsuura, Y., Kikuchi, Y., Hosokawa, T., Koga, R., Meng, X.-Y., Kamagata, Y., ... Fukatsu, T. (2012). Evolution of symbiotic organs and endosymbionts in lygaeid stinkbugs. The ISME Journal, 6(2), 397-409. doi: 10.1038/ismej.2011.103

Matsuura, Y., Moriyama, M., Łukasik, P., Vanderpool, D., Tanahashi, M., Meng, X.-Y., ... Fukatsu, T. (2018). Recurrent symbiont recruitment from fungal parasites in cicadas. Proceedings of the National Academy of Sciences, 115(26), E5970-E5979. doi: 10.1073/pnas.1803245115

McCutcheon, J. P., Boyd, B. M., \& Dale, C. (2019). The Life of an Insect Endosymbiont from the Cradle to the Grave. Current Biology: CB, 29(11), R485-R495. doi: 10.1016/j.cub.2019.03.032 
McCutcheon, J. P., \& Moran, N. A. (2012). Extreme genome reduction in symbiotic bacteria. Nature Reviews Microbiology, 10(1), 13-26. doi: 10.1038/nrmicro2670

Meibom, K. L., \& Charbit, A. (2010). The unraveling panoply of Francisella tularensis virulence attributes. Current Opinion in Microbiology, 13(1), 11-17. doi: 10.1016/j.mib.2009.11.007

Moran, N. A. (1996). Accelerated evolution and Muller's rachet in endosymbiotic bacteria. Proceedings of the National Academy of Sciences of the United States of America, 93(7), 2873-2878. doi: 10.1073/pnas.93.7.2873

Moran, Nancy A., McCutcheon, J. P., \& Nakabachi, A. (2008). Genomics and Evolution of Heritable Bacterial Symbionts. Annual Review of Genetics, 42(1), 165-190. doi: 10.1146/annurev.genet.41.110306.130119

Moran, Nancy A., Tran, P., \& Gerardo, N. M. (2005). Symbiosis and insect diversification: an ancient symbiont of sap-feeding insects from the bacterial phylum Bacteroidetes. Applied and Environmental Microbiology, 71(12), 8802-8810. doi: 10.1128/AEM.71.12.8802-8810.2005

Nakabachi, A., Yamashita, A., Toh, H., Ishikawa, H., Dunbar, H. E., Moran, N. A., \& Hattori, M. (2006). The 160-kilobase genome of the bacterial endosymbiont Carsonella. Science (New York, N.Y.), 314(5797), 267. doi: 10.1126/science.1134196

Nurk, S., Meleshko, D., Korobeynikov, A., \& Pevzner, P. A. (2017). metaSPAdes: a new versatile metagenomic assembler. Genome Research, 27(5), 824-834. doi: 10.1101/gr.213959.116

Oliver, K. M., Russell, J. A., Moran, N. A., \& Hunter, M. S. (2003). Facultative bacterial symbionts in aphids confer resistance to parasitic wasps. Proceedings of the National Academy of Sciences of the United States of America, 100(4), 1803-1807. doi: 10.1073/pnas.0335320100

Olivieri, E., Epis, S., Castelli, M., Varotto Boccazzi, I., Romeo, C., Desirò, A., ... Sassera, D. (2019). Tissue tropism and metabolic pathways of Midichloria mitochondrii suggest tissue-specific functions in the symbiosis with Ixodes ricinus. Ticks and Tick-Borne Diseases. doi: 10.1016/j.ttbdis.2019.05.019 
Price, M. N., Dehal, P. S., \& Arkin, A. P. (2009). FastTree: computing large minimum evolution trees with profiles instead of a distance matrix. Molecular Biology and Evolution, 26(7), 16411650. doi: $10.1093 / \mathrm{molbev} / \mathrm{msp} 077$

Ramaiah, A., \& Dasch, G. A. (2018). Genome Sequence of Coxiella-Like Endosymbiont Strain CLERmD, a Bacterial Agent in the Cattle Tick, Rhipicephalus microplus Deutsch Strain. Genome Announcements, 6(13). doi: 10.1128/genomeA.00003-18

Rispe, C., \& Moran, N. A. (2000). Accumulation of Deleterious Mutations in Endosymbionts: Muller's Ratchet with Two Levels of Selection. The American Naturalist, 156(4), 425-441. doi: $10.1086 / 303396$

Ronquist, F., Teslenko, M., van der Mark, P., Ayres, D. L., Darling, A., Höhna, S., ... Huelsenbeck, J. P. (2012). MrBayes 3.2: Efficient Bayesian Phylogenetic Inference and Model Choice Across a Large Model Space. Systematic Biology, 61(3), 539-542. doi: 10.1093/sysbio/sys029

Rowe, H. M., \& Huntley, J. F. (2015). From the Outside-In: The Francisella tularensis Envelope and Virulence. Frontiers in Cellular and Infection Microbiology, 5, 94. doi: 10.3389/fcimb.2015.00094

Russell, J. A., Goldman-Huertas, B., Moreau, C. S., Baldo, L., Stahlhut, J. K., Werren, J. H., \& Pierce, N. E. (2009). Specialization and geographic isolation among Wolbachia symbionts from ants and Iycaenid butterflies. Evolution; International Journal of Organic Evolution, 63(3), 624-640. doi: 10.1111/j.1558-5646.2008.00579.x

Sachs, J. L., Skophammer, R. G., \& Regus, J. U. (2011). Evolutionary transitions in bacterial symbiosis. Proceedings of the National Academy of Sciences, 108(Supplement 2), 10800-10807. doi: 10.1073/pnas.1100304108

Scarborough, C. L., Ferrari, J., \& Godfray, H. C. J. (2005). Aphid protected from pathogen by endosymbiont. Science (New York, N.Y.), 310(5755), 1781. doi: 10.1126/science.1120180 
Scott, J. D., \& Durden, L. A. (2015). Amblyomma dissimile Koch (Acari: Ixodidae) parasitizes bird captured in Canada. Systematic and Applied Acarology, 20(8), 854-860. doi: 10.11158/saa.20.8.2

Shivaprasad, H. L., Cadenas, M. B., Diab, S. S., Nordhausen, R., Bradway, D., Crespo, R., \& Breitschwerdt, E. B. (2008). Coxiella-like infection in psittacines and a toucan. Avian Diseases, 52(3), 426-432. doi: 10.1637/8192-120707-Reg

Smith, T. A., Driscoll, T., Gillespie, J. J., \& Raghavan, R. (2015). A Coxiella-Like Endosymbiont Is a Potential Vitamin Source for the Lone Star Tick. Genome Biology and Evolution, 7(3), 831838. doi: 10.1093/gbe/evv016

Sudakaran, S., Kost, C., \& Kaltenpoth, M. (2017). Symbiont Acquisition and Replacement as a Source of Ecological Innovation. Trends in Microbiology, 25(5), 375-390. doi: 10.1016/j.tim.2017.02.014

Takiya, D. M., Tran, P. L., Dietrich, C. H., \& Moran, N. A. (2006). Co-cladogenesis spanning three phyla: leafhoppers (Insecta: Hemiptera: Cicadellidae) and their dual bacterial symbionts. Molecular Ecology, 15(13), 4175-4191. doi: 10.1111/j.1365-294X.2006.03071.x

Theis, K. R., Dheilly, N. M., Klassen, J. L., Brucker, R. M., Baines, J. F., Bosch, T. C. G., ... Bordenstein, S. R. (2016). Getting the Hologenome Concept Right: an Eco-Evolutionary Framework for Hosts and Their Microbiomes. MSystems, 1(2), e00028-16. doi: 10.1128/mSystems.00028-16

Thompson, J. D., Gibson, T. J., \& Higgins, D. G. (2002). Multiple sequence alignment using ClustalW and ClustalX. Current Protocols in Bioinformatics, Chapter 2, Unit 2.3. doi: 10.1002/0471250953.bi0203s00

Turner, T. R., James, E. K., \& Poole, P. S. (2013). The plant microbiome. Genome Biology, 14, 209. doi: 10.1186/gb-2013-14-6-209

van Schaik, E. J., Chen, C., Mertens, K., Weber, M. M., \& Samuel, J. E. (2013). Molecular pathogenesis of the obligate intracellular bacterium Coxiella burnetii. Nature Reviews. Microbiology, 11(8), 561-573. doi: 10.1038/nrmicro3049 
Vapniarsky, N., Barr, B. C., \& Murphy, B. (2012). Systemic Coxiella-like infection with myocarditis and hepatitis in an eclectus parrot (Eclectus roratus). Veterinary Pathology, 49(4), 717-722. doi: $10.1177 / 0300985811409251$

Vautrin, E., \& Vavre, F. (2009). Interactions between vertically transmitted symbionts: cooperation or conflict? Trends in Microbiology, 17(3), 95-99. doi: 10.1016/j.tim.2008.12.002

Voordouw, M. J. (2015). Co-feeding transmission in Lyme disease pathogens. Parasitology, 142(2), 290-302. doi: 10.1017/S0031182014001486

Wang, M., Zhu, D., Dai, J., Zhong, Z., Zhang, Y., \& Wang, J. (2018). Tissue Localization and Variation of Major Symbionts in Haemaphysalis longicornis, Rhipicephalus haemaphysaloides, and Dermacentor silvarum in China. Applied and Environmental Microbiology, 84(10). doi: 10.1128/AEM.00029-18

Wernegreen, J. J. (2012). Endosymbiosis. Current Biology: CB, 22(14), R555-561. doi: 10.1016/j.cub.2012.06.010

Wickham, H. (2016). ggplot2: Elegant Graphics for Data Analysis (2nd ed.). Retrieved from https://www.springer.com/gp/book/9783319242750

Woc-Colburn, A. M., Garner, M. M., Bradway, D., West, G., D’Agostino, J., Trupkiewicz, J., ... Nordhausen, R. W. (2008). Fatal coxiellosis in Swainson's Blue Mountain Rainbow Lorikeets (Trichoglossus haematodus moluccanus). Veterinary Pathology, 45(2), 247-254. doi: 10.1354/vp.45-2-247

Wright, C. L., Sonenshine, D. E., Gaff, H. D., \& Hynes, W. L. (2015). Rickettsia parkeri Transmission to Amblyomma americanum by Cofeeding with Amblyomma maculatum (Acari: Ixodidae) and Potential for Spillover. Journal of Medical Entomology, 52(5), 1090-1095. doi: 10.1093/jme/tjv086

Zhong, J., Jasinskas, A., \& Barbour, A. G. (2007). Antibiotic treatment of the tick vector Amblyomma americanum reduced reproductive fitness. Plos One, 2(5), e405. doi: 10.1371/journal.pone.0000405 


\section{Acknowledgements}

831 Financial support was provided by the French government's Programmes Investissement d'Avenir (Laboratoire d'Excellence CEBA, the MicroBIOMES Strategic Project 2016-2018 and the MiTick Annual Project 2016) and recurring funding from CNRS and IRD (OD, MB, DA). FB benefits from a PhD fellowship financed by the CEBA Laboratoire d'Excellence and Montpellier University. We wish to thank the platforms MGX (Montpellier Genomics and Bioinformatics Facility) and GenSeq (Montpellier University), for the technical help and the high-throughput sequencing. We are grateful to the members of Institut Pasteur de Guyane, particularly Benoît de Thoisy and Agathe Chavy, for technical support. Special acknowledgement to the "Agence Nationale de la Preservation de la Nature" (ANPN) and the "Centre National de la Recherche Scientifique et Technologique of Gabon" (CENAREST) that authorized and facilitated the access to the national parks in Gabon, and Marc F. Ngangué (ANPN) that participated to the field collections in Gabon. We also thank Frédéric Delsuc, Xavier Bailly, Laurence Mouton and Christine Chevillon for helpful discussions. The authors acknowledge the IRD itrop HPC (South Green Platform) at IRD Montpellier for providing

845 HPC resources that have contributed to the research results reported in this paper. We also acknowledge useful discussions with members of the Tiques et Maladies à Tiques (TMT) working group from the Réseau Ecologique des Interactions Durables (REID).

\section{Conflict of interest}

850 The authors declare that they have no conflict of interest. 


\section{Figure legends}

852 Figure 1 Phylogenetic relationship of the 25 Amblyomma species examined in this study. The phylogenetic tree was inferred using Bayesian inferences (BI) from a large fragment of rDNA sequences (18S, ITS1, 5.8S, ITS2, and 28S rDNA; 3312 bp unambiguously aligned; best-fit approximation for the evolutionary model: $\mathrm{GTR}+\mathrm{G}+\mathrm{I})$, the numbers on nodes indicate the BI probability. One to two specimens per Amblyomma species (45 specimens in total) were analysed. The vertebrate species on which tick species mainly feed (host-range) is shown. White circles, African group (AF); black circles, New Word group (NW).

Figure 2 Diversity of the microbiome and bacterial prevalence in Amblyomma species. The left part of the Figure shows the Amblyomma phylogenetic tree adapted from Figure 1. One to two specimens per Amblyomma species (45 specimens in total) were included in the phylogenetic analysis. A the middle part, the heatmap shows the abundance and diversity of the nine most abundant OTUs characterized in the $16 \mathrm{~S}$ rRNA bacterial data set for each tick species. The number on the right of the heatmap indicates the number of tick specimens per species used to construct the heatmap (one to ten specimens per Amblyomma species (142 specimens in total) were included in the microbiote analysis). On the right of the figure, the prevalence of the three most common symbionts is illustrated by the coloured squares (Coxiella-LE, blue square; Francisella-LE, green square and Rickettsia, red square): full square indicate a prevalence of $100 \%$, while empty or partly colored squared indicate 871 prevalences from 0 to $<100 \%$ (the numbers besides these squares indicate the prevalences).

872 The amount of DNA for the single specimen of $A$. sculptum was not sufficient to perform the 873 16S rRNA barcoding, therefore the heatmap for this species is not applicable (N/A). In this 874 latter species, the presence of Coxiella-LE and Francisella-LE was asserted through specific 
PCR assays as detailed in the text. White circles, Amblyomma African group (AF); black circles, Amblyomma New Word group (NW).

Figure 3 Structuration of the Amblyomma microbiome. A Multidimensional scaling plot (MDS) of the microbial diversity of each Amblyomma species depending on the presence of both Coxiella-LE (red dots) and Francisella-LE (green dots) or the absence of obligate symbiont (blue dots). B MDS plot of the microbial diversity depending on the presence (red dots) / absence (blue dots) of Rickettsia. C MDS plot of the microbial diversity depending on the association / no association between the three symbionts (Coxiella-LE, Francisella-LE and Rickettsia): C_R (red dots) correspond to Amblyomma specimens co-infected by Coxiella-LE and Rickettsia; C (brownish dots) to specimens only infected by Coxiella-LE; F (green dots) to specimens only infected by Francisella-LE; F_R to specimens co-infected by Francisella-LE and Rickettsia; C_F_R (light blue dots) to specimens multi-infected by Coxiella-LE, Francisella-LE and Rickettsia; dark blue dots to specimens not infected by these symbionts. Each MDS plot is based on the generalized Unifrac $(\alpha=0.5)$ distance matrix and all confidence ellipses are been drawn with the confidence level of 0.95 .

Figure 4 Evolutionary histories between Amblyomma and their nutritional symbionts. A Cophylogeny between the phylogenies of Amblyomma (on the left) and Coxiella-LE (on the right): The tick phylogeny is based on 3312-bp nuclear rDNA sequences (18S, ITS1, 5.8S, ITS2, and 28S rDNA; best-fit approximation for the evolutionary model: GTR+G+I); the Coxiella-LE phylogeny was reconstructed using a concatenated gene sequence (16S rRNA, rpoB and groEL concatenated sequences, 2045 unambiguously aligned base pairs; best-fit approximation for the evolutionary model: GTR+G+I). B Cophylogeny between the phylogenies of Amblyomma (on the left) and Francisella-LE (on the right): The tick 
900 phylogeny is based on 3312-bp nuclear rDNA sequences (18S, ITS1, 5.8S, ITS2, and 28S

901 rDNA; best-fit approximation for the evolutionary model: GTR+G+I); the Francisella-LE 902 phylogeny was reconstructed using a concatenated gene sequence (16S rRNA, rpoB, groEL,

903 fts $Z$ and $g y r B$ concatenated sequences, 3506 unambiguously aligned base pairs; best-fit

904 approximation for the evolutionary model: GTR+G+I). Each phylogeny was reconstructed

905 using BI and node numbers are the posterior probabilities (only values $>70$ are shown). White

906 circles, Amblyomma African group (AF); black circles, New Word group (NW). 